\title{
CMS searches for new physics
}

\section{Mara Senghi Soares*On behalf of the CMS Collaboration}

E-mail: mara.senghi@ciemat.es

We discuss the first searches for new physics being carried out with the CMS detector, at the early stage of data taking. Prospects for discoveries of new exotic and supersymmetric states are discussed. Methods developed for background control in searches for new physics using the very first data of $p p$ collisions at $\sqrt{s}=7 \mathrm{TeV}$ are presented.

Kruger 2010: Workshop on Discovery Physics at the LHC

December 5 - 10, 2010

Kruger National Park, Mpumalanga, South Africa

*Speaker. 


\section{Introduction}

The first $p p$ collision data collected with the CMS detector [1] since the starting of the LHC operation in 2009 revealed an amazing performance of the detector. CMS demonstrated an excellent response both in terms of particle identification and in accuracy of the most important variables describing the $p p$ interaction. A vast range of processes involving leptons, photons, jets and missing transverse energy (MET) was measured with the data collected in 2009 and beginning of 2010. Even at very low integrated luminosities during this LHC start-up period, the data were found to be in very good agreement with the Standard Model (SM) predictions. With a detector fairly well understood and the physics of the SM commissioned, signals compatible with new physics, if existing, may be seen very soon in the CMS data, as the integrated luminosity starts to increase.

We discuss some of the analyses that are part of the extensive program of searches for new physics at CMS. The emphasis here is given to the processes where potential discoveries or improved exclusion limits will be possible with up to $1 \mathrm{fb}^{-1}$ of integrated luminosity at $\sqrt{s}=7 \mathrm{TeV}$, expected to be collected during 2010 and 2011 data-taking periods. Other important searches, not mentioned in this contribution, are discussed in Ref. [2]. Results presented in other CMS contributions to this conference are also not discussed.

We present prospects for discovery or stringent limits setting on exotics and supersymmetric signals based on Monte Carlo simulation studies. Methods based on data [3] for background predictions and identification of prompt and non-prompt leptons are discussed, and results from studies using the very first CMS data at $\sqrt{s}=7 \mathrm{TeV}$ are shown.

\section{Search strategy for new exotic states}

The SM with three generations of quarks sucessfully describes the experimental data in particle interactions known to this date. However, the existence of a fourth generation of massive fermions $\left(b^{\prime}\right)$ has important implications on the Higgs mass determination [4] and could also help explaining the baryon asymmetry of the Universe [5]. For CMS searches [6], the mass of the $b^{\prime}$ quark, produced in pairs, is assumed to be above the sum of the quark top and $\mathrm{W}$ boson masses. In this case, the dominant decay mode is the $t W$ channel, and the full process is $p p \rightarrow b^{\prime} \bar{b}^{\prime} \rightarrow t W^{-} \bar{t} W^{+}$. Taking into account further decays $t \rightarrow W b$ and $\mathrm{W} \rightarrow$ lepton-neutrino or di-jets, the signature for such signal is the presence of two or three leptons of the same sign, accompanied by jets and/or missing transverse energy (MET) in the final state. Since events with same-sign leptons are rare in the SM, this search profits from very small background yields. Figure 1 shows the expected $95 \%$ Confidence Level exclusion limits on the $b^{\prime}$ mass in two scenarios: using samples of integrated luminosities of 200 or $600 \mathrm{pb}^{-1}$, expected to be collected during 2011. Current Tevatron limits on the $b^{\prime}$ mass (>325 GeV) [7] can be surpassed with the integrated luminosity of 2010 only.

Given the striking symmetry observed between the generations of quarks and leptons, it is natural to think of a more fundamental relationship between them, absent in the SM. The existence of particles carrying both baryon and lepton numbers - the leptoquarks - is predicted in several theoretical models. To avoid the creation of flavor-changing neutral currents and lepton family-number violations, leptoquark couplings are restricted within a single lepton-quark generation. Results on the search for leptoquarks of the first generation is presented in another contribution to this con- 
ference. The second generation of leptoquarks [8] would be found in final states containing two high transverse momentum muons and two high transverse momentum jets. The most important SM background competing with this final state are $t \bar{t}$, bosons and dibosons accompanied with jets. Figure 2 shows the leptoquark search sensitivity as a function of its mass and Branching Fraction into a charged lepton, $\beta$.

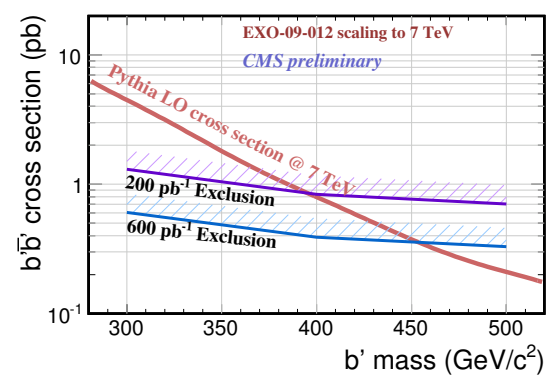

Figure 1: Expected limits on the $b^{\prime}$ mass, at $95 \%$ C.L., for a $7 \mathrm{TeV} \mathrm{LHC}$ run, 200 and $600 \mathrm{pb}^{-1}$.

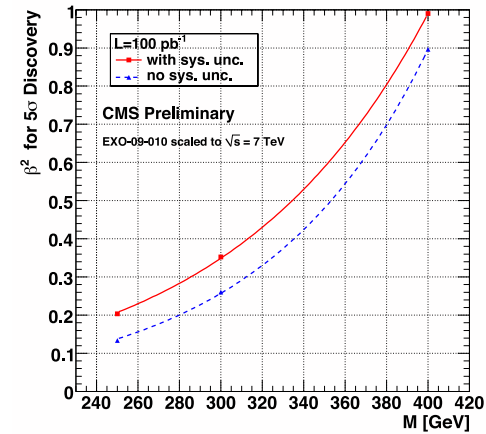

Figure 2: $95 \%$ C.L. for leptoquarks discovery with $100 \mathrm{pb}^{-1}$ at $7 \mathrm{TeV}$. More details in the text.

One of the remaining SM issues is the hierarchy problem: the fundamental scale of gravity $\left(M_{\mathrm{Pl}}\right)$ is many orders of magnitude larger than the electroweak symmetry breaking scale, implying very large radiative corrections on the Higgs boson mass, unless a very fine tuning is performed. A possible solution is found in the context of Extra Dimensions (ED). In the ADD model [9] the SM is constrained to the usual 3+1 space-time dimensions, but gravity is free to propagate to the entire multidimensional space. The fundamental Planck scale $M_{D}$ would scale down with respect to $M_{\mathrm{Pl}}$ through the multidimensional Gauss law: $M^{\mathrm{n}_{\mathrm{ED}}+2}=M_{\mathrm{Pl}}^{2} / r^{\mathrm{n}_{\mathrm{ED}}}$, where $r$ and $\mathrm{n}_{\mathrm{ED}}$ are the size and number of the large extra dimensions (LED), respectively. In the Randall-Sundrum (RS) model [10], the observed hierarchy is created by a warped extra dimension and the gravitons appear as a tower of Kaluza-Klein excitations. Signals of ED could be observed in several final states [11]. Real graviton production $(q \bar{q} \rightarrow g G, q g \rightarrow q G$ and $g g \rightarrow g G)$ would be characterized by the observation of a Mono-Jet final state: only one single jet at very high- $p_{T}$, compensated by the same amount of MET. LED via virtual graviton exchange could be detected on the diphoton channel, as an excess of events on the tail of the invariant mass of the diphoton system $M_{\gamma \gamma}$, above the SM predictions. The same experimental data could be used to impose limits on RS gravitons, but in this case a narrow resonance would be observed in the $M_{\gamma \gamma}$ spectrum instead of a flat excess of events. Expected limits for various hypothesis on the luminosity, scale and number of ED are displayed in Figure 3.

\section{Search strategy for Supersymmetry (SUSY)}

The search for supersymmetric particles is an important part of the CMS program on new physics. The strategy consists in designing analyses that are not constrained to one single model (nor to a set of models), keeping the searches as generic as possible. The completely modelindependent approach limits the estimation of the sensitivity on SUSY searches at CMS. The lack of full knowledge on the backgrounds and their experimental uncertainties, discussed in the next section, also imposes some limitation. Here, we express the search sensitivity in terms of the 

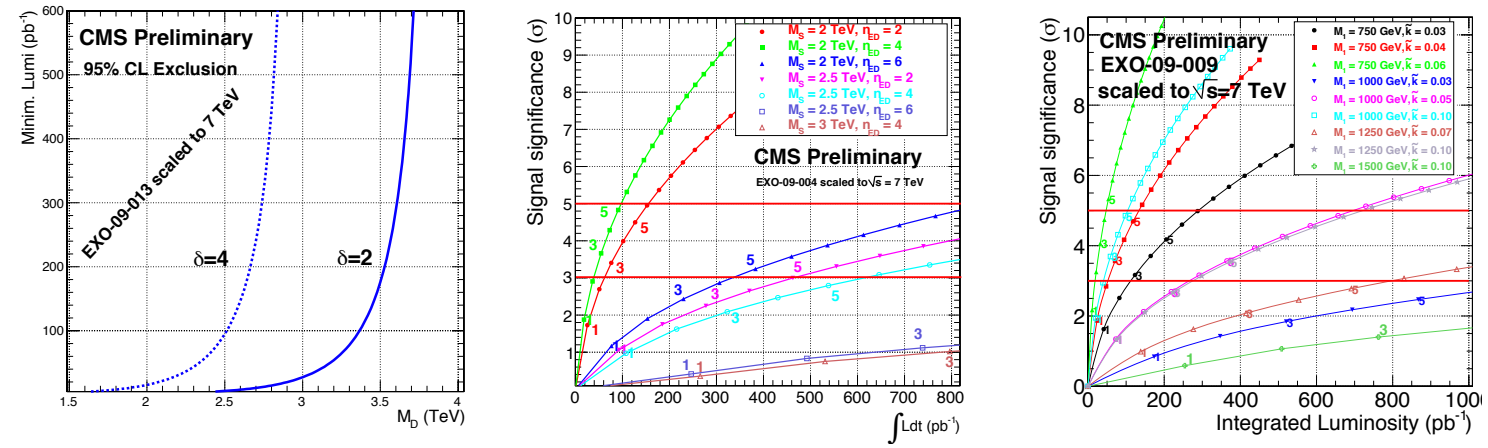

Figure 3: Expected exclusion limits on ED, at 95\% C.L.. Left: LED in the Mono-Jet channel for $n_{\mathrm{ED}} \equiv$ $\delta=2$, 4; center: LED in diphoton channel; right: RS gravitons in the diphoton channel, as a function of the model parameters.

parameters of the commonly used benchmark mSUGRA [12], in two final states: the all-hadronic channel and the like-sign dilepton channel. The estimation, shown in Figure 4, uses background prediction from simulated events. Only $100 \mathrm{pb}^{-1}$ of integrated luminosity are needed for CMS to surpass current Tevatron and LEP limits on a large region of the mSUGRA parameter space.
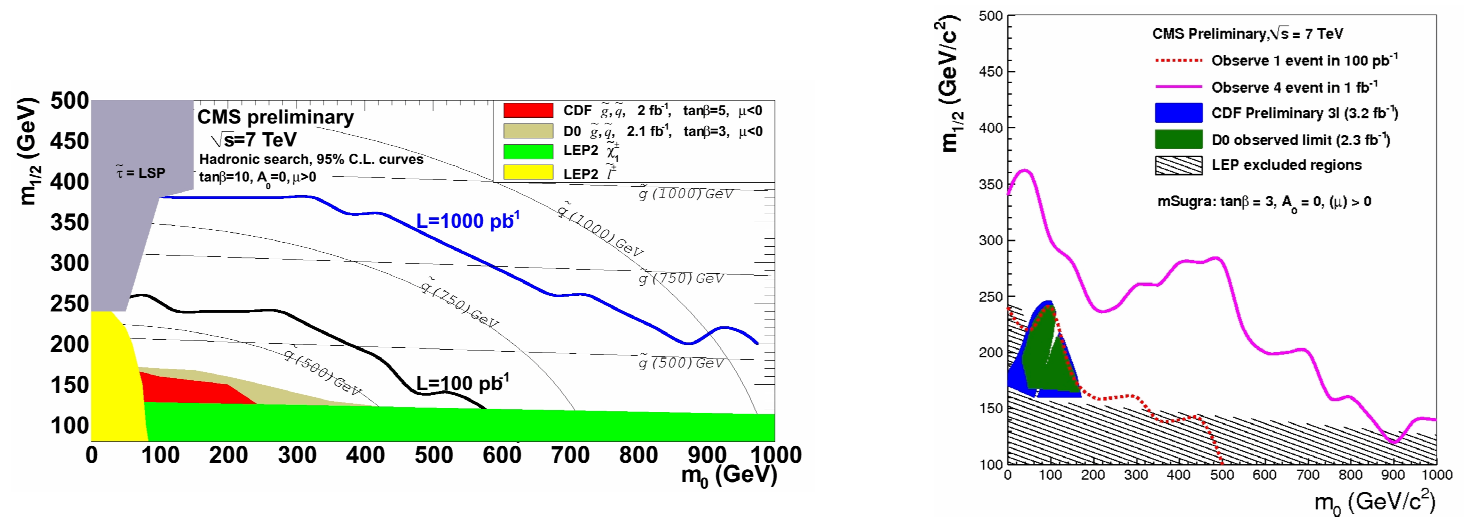

Figure 4: Expected limits, at 95\% C.L., for all-hadronic (left) and like-sign dileptons (right) channels as functions of mSUGRA parameters.

\section{Methods for background controlling in searches with the early data}

Understanding the background is fundamental on the search for new physics. Signals of new physics, usually characterized by unknown or very small cross-sections, must be unmistakably well separated from the SM background. Specially dangerous are the backgrounds from SM processes that are still not known to a very high precision, or from mismeasurements on important objects such as jets, leptons and MET. Backgrounds of this nature have to be estimated directly from the data. Although not the dominant source, QCD background estimation is critical due to the lack of higher-order corrections on theoretical calculations. Besides the uncertainty on the normalization of the cross-section prediction, topological searches are highly affected, since in QCD basic quantities such as the number of jets or the jet $p_{T}$ distributions can not be precisely predicted.

An important variable for QCD control is $\alpha_{T}$, which characterizes the overall transverse momentum balance of the event. For a dijet system, it can be defined in terms of the well known 


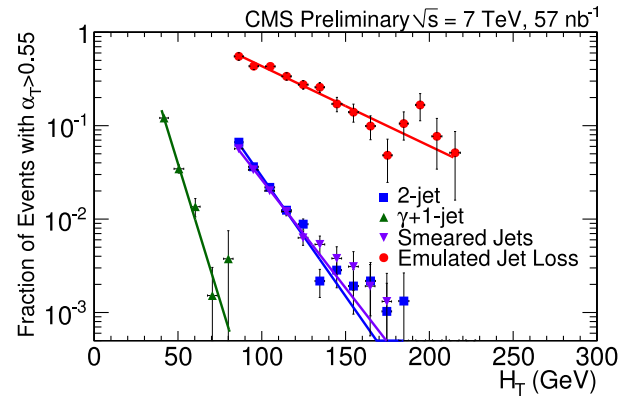

Figure 5: Fraction of events with $\alpha_{T}>0.55$ cut as a function of $H_{T}$, for samples of di-jet, $\gamma+$ jet samples and emulated jet loss and smearing.

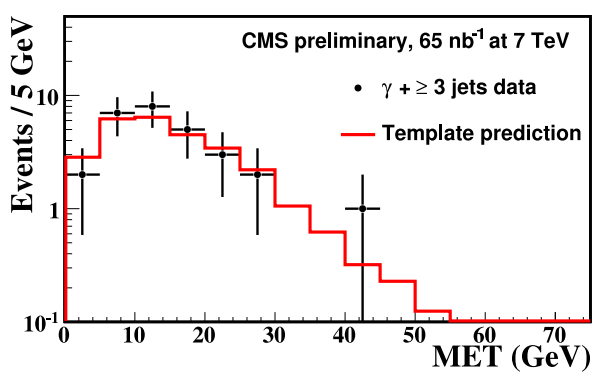

Figure 6: Artificial MET in early CMS data: comparison between predicted distribution from templates in QCD events and measured in $\gamma+$ jets events

quantities $H_{T}$ and $\mathrm{MHT}^{1}$ as $\alpha_{T}=\frac{1}{2} \frac{H_{T}-\Delta H_{T}}{\sqrt{H_{T}^{2}-(\mathrm{MHT})^{2}}}$. In a genuine QCD event where no real MET is present, $\alpha_{T}=0.5$ if the two jets are perfectly balanced and $<0.5$ if one jet is mismeaured. However, in the presence of MET - either real from non-QCD events or "fake" as discussed next, $\alpha_{T}$ assumes larger values. Vetoing events with $\alpha_{T}<0.55$ should cut off the bulk of QCD events since $\alpha_{T}$ distribution falls sharply at $\lesssim 0.5$, but a residual tail will be present. The fraction of events with $\alpha_{T}>0.55$ in a sample of well measured QCD events is a good estimation of the further QCD contamination on events with fake MET. The important feature of this method is that this fraction always decreases as $H_{T}$ increases. This is shown in Figure 5 for samples of dijets, $\gamma+$ jet and for artificially degradated data, simulating very poor jet reconstruction. Therefore, an estimation of the QCD contribution at a given $H_{T}$ can be safely extrapolated as an upper limit to the region of interest at high- $H_{T}$, where SUSY signals are expected to appear, and where it is extremely difficult to estimate QCD background from the data due to the lack of statistics.

Events with a large amount of MET are also very important in SUSY searches, since the background from SM processes involving neutrinos is much reduced and can be more precisely determined. Exotic searches as the Mono-Jet channel also depend on precise MET determination. On the experimental side, however, MET measurements can be quite challenging, demanding a very accurate reconstruction of the complete event. Some sources of artificial MET, such as imperfect event reconstruction due to software effects or instrumental response, or the presence of non-collision backgrounds, can produce tails on the MET distributions. This "fake" MET competes with the signals containing genuine MET expected in supersymmetric processes, and can not be estimated from simulation only. Here, we use samples of QCD events - where no real MET is present - to model the artificial MET production. Templates are build to measure the amount of artificial MET that is produced as fuctions of $H_{T}$ and the number of jets. The model was tested by comparing the template predictions to the MET measured in photon+jets events, another sample where real MET is also not present. Results are shown in Figure 6. Very good agreement between measurement and prediction is found. The predictions from the templates are then used to estimate the artificial MET contaminating real MET in SUSY and exotic searches.

\footnotetext{
${ }^{1}$ SUSY events are expected to be found at very high values of $H_{T}=\sum_{\text {jets }(j)} p_{T(j)}$. Missing $H_{T}$ is defined as MHT $=\left|\sum_{\text {jets }(j)}-\vec{p}_{T(j)}\right|$ and $\Delta H_{T} \equiv p_{T 1}-p_{T 2}$, where the jet index obeys the ordering in $p_{T}$.
} 


\section{Conclusions}

In this talk we revised some of the important searches for new physics that are part of the CMS program with early data. Prospects for finding new exotic and supersymmetric particles, based on simulation predictions, were presented. With the first LHC data already available for analysis, at the same time as the searches are being carried out, an exhaustive work on background estimation is being performed. Methods based on data were shown to be powerful to estimate and control different sources of background, which is fundamental to improve searches sensitivity. The studies shown here are just a small fraction of the extensive CMS physics program being developed. They show that CMS is in an excellent position to discover signals of new physics, or at least to impose more stringent limits where searches for exotic and supersymmetric were unsuccessful to this date, in some cases with even less than $1 \mathrm{fb}^{-1}$ of data.

\section{References}

[1] CMS Collaboration, The CMS experiment at the CERN LHC, JINST 3(2008) S08004.

[2] CMS Collaboration, The CMS physics reach for searches at $\sqrt{s}=7 \mathrm{TeV}$, CMS Note 2010/008.

[3] CMS Coll.,Performance of Methods for Data-Driven Background Estimation in SUSY Searches, CMS-PAS-SUS-10-001.

[4] P.H.Frampton, P.Q.Hung and M.Sher,Quarks and leptons beyond the third generation, Phys. Rept. 330 (2000) 263; G.D.Kribs et al., Four generations and Higgs physics, Phys. Rev. D76 (2007) 075016.

[5] W.-S.Hou, CP Violation and Baryogenesis from New Heavy Quarks, Chin. J. Phys. 47 (2009) 134.

[6] CMS Coll., Search for A Fourth Generation b Quark in tW Final State at CMS in pp Collisions at $\sqrt{s}$ $=10 \mathrm{TeV}, \mathrm{CMS}-\mathrm{PAS}-\mathrm{EXO}-09-012$.

[7] CDF Coll.,Search for Fermion-Pair Decays $Q \bar{Q} \rightarrow\left(t W^{\mp}\right)\left(\bar{t} W^{ \pm}\right)$in Same-Charge Dilepton Events, Phys. Rev. Lett. 104 (2010) 091801.

[8] CMS Coll.,Search for 2nd Generation Scalar Leptoquarks with the CMS Detector, CMS-PAS-EXO-09-010.

[9] N. Arkani-Hamed, S. Dimopoulos, and G. Dvali, The Hierarchy problem and new dimensions at a millimeter, Phys. Lett. B429 (1998) 263; N. Arkani-Hamed, S. Dimopoulos, and G. Dvali, Phenomenology, astrophysics and cosmology of theories with submillimeter dimensions and TeV scale quantum gravity, Phys.Rev. D59 (1999) 086004.

[10] L. Randall, R. Sundrum, Large Mass Hierarchy from a Small Extra Dimension, Phys. Rev. Lett. 83 (1999) 3370 and ibid (1999) 4690.

[11] CMS Coll., Search for Mono-Jet Final States from ADD Extra Dimensions, CMS-PAS-EXO-09-013; Search for Large Extra Dimensions in the Diphoton Final State, CMS-PAS-EXO-09-004; Search for Randall-Sundrum Gravitons in the Diphoton Final State, CMS-PAS-EXO-09-009.

[12] A.Chamseddine, R.Arnowitt and P.Nath, Locally Supersymmetric Grand Unification, Phys. Rev. Lett. 49 (1982) 970; E.Cremmer, P.Fayet, and L.Girardello, Gravity Induced Supersymmetry Breaking and Low-Energy Mass Spectrum, Phys. Lett. B 122 (1983) 41. 